\title{
LE NOM DE L'ARAIGNÉE EN IRLANDE.
}

Étude de sémantique.

\section{I.}

En lisant la Grammaire du Vieil-Irlandais de M. J. Vendryès (Paris, 1908), si remarquable à tant d'égards, je suis resté pensif au passage suivant, p. 116, $\S 210$, dı chapitre des adjectifs:

'Au génitif pluriel, à côté de la forme à désinence -e $n$, existe une forme plus courte, caractérisée seulement par la position antérieure de la consonne. Ainsi: inna damán $n$-allaid, 'des araignées', Ml. $59 \mathrm{~d} 1$, à côté de inna n-dam n-altae, 'des bœufs sauvages', 121 c 19.

Comment le même nom d'animal - en suppossant que ce soit deux fois le même mot - peut-il - et à une ligne de distance! - se traduire successivement par 'araignée' et par 'bœuf sauvage'? Cette question de sêmantique n'a pas préoccupé M. Vendryès, parce que pour lui, linguiste, il importe surtout de noter si telle consonne de tel mot est, dans la cavité buccale, ce qu'il appelle de position ou antérieure ou postérieure. - Puis, lorsqu'il s'agit d'un terme composé, composé d'un substantif et d'un adjectif, pourquoi M. Vendryès n'a-t-il traduit l'adjectif que dans le second cas? Car, si le bœuf est ici - grammaticalement - 'sauvage', l'araignée l'est également, même du point de vue grammatical. - Et enfin, que peuvent être, en dehors d'une grammaire et dans la réalité, des 'bœufs sauvages'? A supposer qu'on puisse donner ce nom, par extension et par analogie, à des animaux comme l'urus de l'ancienne Germanie, l'aurochs de la Lithuanie, le buffle de l'Amérique du Sud, le yali du Tibet, ces animaux n'ont jamais existé en Irlande. Et un bœuf s'échappât-il de la servitude, il ne pourrait devenir sauvage et vivre à l'état 
de liberté comme le chat, le chien ou le cheval, car il lui manque par définition (je pourrais dire: par soustraction) les sources de l'énergie et de la volonté. Bien loin d'être 'sauvage', le bœuf est un être de civilisation: l'animal ne nait pas tel, il le devient par la main de l'homme.

Voulant tirer au clair cette question de zoologie linguistique, je me suis d'abord reporté au texte des gloses citées par M. Vendryès, pour me rendre compte des termes latins que les mots irlandais traduisent. Dans le premièr cas (Ml. $59 \mathrm{~d} 1$ ) il y a bien aranearum, mais dans le second (Ml. $121 \mathrm{c} 19)$ il y a cervos, ce qui ne peut se traduire, en français même poétique, par 'bœufs sauvages'. J'en appelle au témoignage des dictionnaires latins et au jugement des latinistes. 'Sauvage' est une épithète qui peut, avec droit, s'appliquer au cerf, car le cerf est, par nature, un animal sauvage, tandis que le boeuf est un animal domestique.

Mais le problème n'est que déplacé, car il faut maintenant expliquer comment le même nom a pu désigner, en même temps, l'araignée et le cerf.

\section{II.}

Il est aisé de voir que le terme irlandais est formé de deux mots, du substantif dam et de l'adjectif allaid. Ce dernier signifie 'sauvage', non pas au sens de fauve ou d'ennemi de l'homme, mais silvaticus, vivant loin de l'homme, puis, par extension, non apprivoisé, non domestiqué. L'adjectif sert donc de 'déterminant' ou 'exposant' quand il est joint au nom d'un animal, et qu'il l'oppose ainsi à l'animal que l'homme a soumis ou qu'il connait déjà. C'est ainsi que pour les Irlandais le loup est 'le chien sauvage'.

C'est un procédé naturel et ordinaire en psychologie de nommer un objet inconnu par le nom d'un objet déjà connu, nom auquel on ajoute un 'déterminant' pour le différencier. Ainsi, lorsque les Romains virent pour la première fois des éléphants dans l'armée de Pyrrhus, et cela en Lucanie, région de l'Italie du Sud, ils les appelèrent 'boeufs de Lucanie', Lucae boves. De même, on assure que lorsque les habitants de l'extrême nordouest de l'Europe, Lapons ou Finnois, virent pour la première 
fois des boeufs, ils appelèrent ceux-ci du nom qu'ils donnaient $\grave{a}$ leurs rennes. 1)

En ce qui concerne l'emploi du 'déterminant' irlandais allaid 'sauvage' nous ne pouvons mieux résumer la question qu'en citant M. Wh. Stokes dans sa préface aux Lives of Saints from the Book of Lismore (Oxford, 1890, p. xcIv): 'Wild animals are the stag (ag allaid, dam allaid or oss allaid) where the adjective allaid 'wild', is added to the name of a bovine animal (oss, gen. oiss, cognate with Skr. alihsan-, Goth. auhsa), in-t-agh, ocdam. For deer, the word is fiad. The name of wolf, cú allaid, pl. coin allta is, like those of stag, formed with the aid of the adjective allaid'.

Le mot dam signifie, grosso modo 'bœuf'; mais sans doute il avait, à une époque ancienne, le sens plus général de 'quadrupède apprivoisé'. Pour les Irlandais qui s'en servaient au labour, qui se nourrissaient de son lait et de sa chair, pour lesquels la vache était une valeur d'échange et une sorte de 'monnaie de compte', le bœuf était l'animal domestique par excellence. Mais on ne peut oublier qu'au même radical dam appartient le gallois dafad 'a sheep, a ewe' et le breton-armoricain danfad 'brebis'. On ne peut pas oublier non plus notre français daim qui vient d'un bas-latin damus, et celui-ci est le gallo-latin dama ou damma. Nous pouvons dire gallo-latin, car le mot ne s'est conservé que dans les dialectes de France: les formes italienne et espagnole sont, nous dit M. Körting ${ }^{2}$ ), empruntées au français. Et l'ancien gallo-latin, dans de nombreux exemples réunis par $\mathbf{M}$. A. Holder ${ }^{3}$, parait bien avoir désigné le chamois. Il faut même encore élargir ce domaine géographique pour citer les mots grees

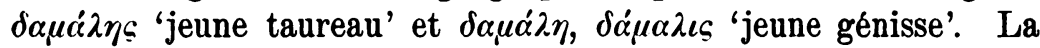
définition étymologique du mot doit donc couvrir et englober les sens, aujourdhui différents, des mots irlandais, gallois, bretons, latins, français et grecs.

A l'occasion du breton danvad (gallois dafad) M. V. Henry ${ }^{4}$ ) a revendiqué l'étymologie par la racine qui se présente aussitôt

1) O. Schrader, Reallexicon etc., Strafsburg, 1901, p. 374, avec référence à Müllenhoff, Deutsche Altertumskunde, II, 356 .

2) Lateinisch-romanisches Wörterbuch, 30 Edition, 1907, s. v. dāmŭs.

3) Alt-Celtischer Sprachschatz. 8. v.

4) Dictionnaire étymologique du breton moderne, Rennes, 1900, p. 87 et 104 . 
à l'esprit des linguistes. C'est, dit-il, le mot correspondant à un celtique *dama-tó, 'apprivoisé, doux', par suite 'mouton', lequel

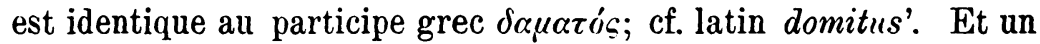
peu plus loin, sous le mot don=gallois dof 'apprivoisé, doux', il ajoute: 'soit un celtique dom-o, vieil-islandais tam-r, angl. tame, all. $z a h m$, qui se rattache à la même racine que le latin dom-āre'. M. A. Walde, dans son récent Lateinisches etymologisches Wörterbuch, Heidelberg 1906, s. v. dāma, n'admet pas cette explication (unwahrscheinlich, dit-il) et il veut voir, à l'origine, le sens de 'bêtes à cornes', mais sans s'expliquer à cet égard; il y aurait d'après lui, mélange (je dirais hybridation) de deux racines. ${ }^{1}$ ) Il nous parait bien plus vraisemblable qu'après avoir eu le sens général d'animal dompté, apprivoisé, domestiqué, le mot a passé avec un 'déterminant' ou épithète, à des animaux que l'homme connaissait et chassait; puis, par abréviation, comme il arrive souvent dans l'histoire des langues (par exemple, en français poulain pour *pullanus equinus) le déterminant est tombé, et le substantif, resté seul, a été fixé dans un sens dérivé et particulier.

\section{III.}

Nous avons donc en irlandais les termes: dam 'bœuf', d'où ro.dam 'grand bœuf', et dam allaid 'cerf', litt. 'dam sauvage'. Comment le même nom a-t-il pu s'appliquer à l'araignée?

Il convient d'abord d'observer que dans le manuscrit de Milan, quand il s'agit de traduire le latin aranea, le glossateur écrit damán - et non pas dan - allaid. M. Vendryès n'a pas remarqué la longueur de l'á final, exprimée par l'accent. Il s'agit donc là d'un diminutif en -án, comme les substantifs si nombreux de cet ordre: M. Vendryès les a lui-même étudiés un peu plus loin, p. 161, § 306, mais il lui a échappé que damán devait figurer dans cette liste. - M. Kuno Meyer, dans ses Contributions to Irish Lexicography donne le mot (s. v.) à la fois comme substantif isolé qu'il traduit 'a calf, or fawn', et comme nom d'homme.

Damán allaid: ce nom qui était celui de l'araignée au VIIIe siècle de notre ère, l'est resté comme on peut voir par les

1) P. S. Je vois que M. Thurneysen adhère à l'explication par la même racine que le latin domare dans son Handbuch des Alt-Irischin, Heidelberg 1909 , p. $114, \S 188$. 
textes et les dictionnaires, et il est, en quelque sorte, la larve d'où est sorti le nom actuel de l'araignée dans la langue parlée. Nous sommes forcé de multiplier les exemples pour montrer la genèse de cette transformation.

M. Kuno Meyer, dans ses Contributions etc., réfère à trois exemples: la glose de Milan; - une citation dans Standish H. O'Grady, Catalogue of Irish Mss. p. 182; - et un texte des Transactions of the Ossianic Society, T. V., 1860, p. 42. - C'est le terme employé dans la traduction (protestante) de la Bible, connue sous le nom de Bedel: Job, VIII, 14, Isaie, LIX, 5. Mac Curtin dans son English-Irish Dictionary, publié à Paris en 1732, donne l'article: Spider, s. darián calla i. peist.1) Les autres dictionnaires, O'Brien, O'Reilly répètent le même mot, comme aussi les dictionnaires postérieurs, puisqu'un dictionnaire est fait d'ordinaire avec ceux qui l'ont précédé, parcè (plus ou moins!) detorta. ${ }^{2}$ ) Mais ils donnent en même temps des variantes où l'on peut voir qu'au cours des temps le mot s'est déformé et transformé.

Nous avons déjà, grace à John K'eogh une forme phonétique de ce nom recueillie voici bientôt deux siècles. John K'eogh était originaire du Sud de l'Irlande: il ne connaissait sans doute pas l'irlandais par les livres, mais par l'usage, et à ce titre, les noms irlandais qu'il cite, pour l'identification, dans sa Botanologia et dans sa Zoologia sont intéressants pour les philologues, en même temps que ses remarques le sont quelquefois pour les folkloristes. Il vaudrait la peine de relever, en forme de liste, pour cette revue ou pour Ériu, les noms de plantes et d'animaux

1) Nous ne comprenons pas bien ce que vient faire ici cette explication additionnelle: .i. peist, car ce mot a, d'ordinaire, le sens de 'monstre', et il ne figure dans ce méme dictionnaire de Mac Curtin, ni sous beast, ni sous animal, ni sous monster.

3) En voici un exemple. O'Reilly contient cet article: damh-allta, s. a wild ox, a buffalo. Buffalo est d'autant moins à sa place que c'est le nom anglais d'un buffle exotique: de plus 'wild ox' est l'explication littérale des termes composant le nom, nullement la traduction. M. Skeat a parlé un jour de ghost-words; ici c'est ce qu'on pourrait appeler un ghost-meaning. - A la suite d'O'Reilly, et par ce qu'O'Reilly contenait cet article, le P. Dinneen a donné celui-ci : Damh allaidh (damh allta) a wild ox, a buffalo, an elk, a roebuck. Au buffalo le P. Dinneen a ainsi ajouté l'élan (Cervus alces) qui n'a existé en Irlande qu'à une époque préhistorique, de même qne le cerviıs megaceros hibernicus. 
présentés par John K'eogh comme traduction des mots anglais. Pour nous en tenir à l'araignée, dans sa Zoologia Medicinalis Hibernica publiée à Dublin en 1739, K'eogh donne, p. 82, duanalla.

Duanalla: voilà ce qu'est devenu, au bout de mille ans, damán allaid. La consonne $m$, étant entre voyelles, est tombée, et a fini par donner lieu à un simple hiatus. Puis, comme en irlandais, les voyelles de même ordre, c. à d. larges ou minces, sont peu distinctes et se confondent volontiers, on a eu des formes avec $u$ radical au lieu d'a; et comme le sens initial diminutif de dam - était oublié, et que l'irlandais écrit, entre voyelles, des consonnes qui ne se prenoncent pas (pour marquer une diérèse historique et traditionnelle), $b h$ a dans l'orthographe, remplacé souvent $m h$. De cette façon, les dictionnaires nous donnent aujourdhui les formes concurrentes - mais concurrentes seulement en apparence, car la prononciation est la même damhán, dabhán et enfin dubhán.

J. O'Brien (je cite d'aprés la seconde édition de 1832) a l'article 'Damhán alla, a spider; potius dubhán alla'. O'Reilly, en 1817, donne, à la fois, damhán allaidh et dubhán alla. Foley (English-Irish Dictionary, Dublin 1855) a: dabhán alla. Le P. Dinneen (en 1904) donne également damhán alla et dubhán alla; et il ajoute, sous ce dernier terme: 'See dabhán alla' le dernier terme est resté dans l'encrier du P. Dinneen, car il manque au dictionnaire: mais on voit, par la citation, que l'auteur l'avait in petto, et que pour lui dabhán, damhán et dubhán, suivis du déterminant alla 'sauvage' sont seulement des prononciations un peu différentes du nom de l'araignée. - Tout récemment encore, M. T. O'Neill Lane (English-Irish Dictionary, Dublin, 1904), nom offre l'article: Spider, dubhán alla, damhán alla. - En somme, les Irlandais ne reconnaissent plus le radical dam 'bœuf' dans leur nom de l'araignée.

Malgré la renaissance de la langue irlandaise, et le renouveau des études celtiques en Irlande, on ne s'est pas occupé de la langue parlée et réelle, au point de vue de la lexicographie. On étudie les mots au point de vue de la phonétique ou de la grammaire, pas au point de vue de la sémantique. Quand on désire connaître la forme réelle du nom d'une plante ou d'un animal, il n'y a guère que les listes très sommaires de J. K'eogh d'il y a deux siècles. Les glossaires sont faits avec les livres, ou dans 
l'orthographe rectifiée des livres et l'amas de consonnes qui ne se prononcent pas; ils ne donnent pas non plus les usages, pratiques et superstitions populaires qui souvent, seules, expliquent le nom d'un animal, ou d'une plante, ou d'un minéral. Il n'y a pas pour l'Irlande d'ouvrage qui ressemble à ce que M. Rolland a fait pour la France et nos pays voisins sous le nom de Faune populaire et Flore populaire, ouvrages rédigés d'après la classification de l'histoire naturelle et où, par conséquent, chaque nom est scientifiquement identifié et précisé. Ce qu'on appelle en anglais, je crois, les words of mouth, manquent au philologue qui étudie les mots celtiques et qui cherche dans ces mots l'histoire des idées ou des choses, non pas la formation physiologique des phonèmes. ${ }^{1}$ )

J'aurais voulu avoir les noms réels et vivants de l'araignée - et même des différentes espèces d'araignées - en Irlande. J'ai seulement pu apprendre, grace à M. l'abbé Patrice 0' Gorman, qu'en Munster dubhán-alla est la forme généralement connue, et que le second terme est compris comme étant le génitif de falla 'mur'. Le premier terme, lui-même, semble se rattacher à $d u b h$ 'noir', comme l'indique déjà la graphie adoptée (on vient de la voir) dans les dictionnaires actuels. Dans la conscience de ceux qui parlent aujourd'hui irlandais, le nom de l'araignée signifie donc 'la noiraude du mur': c'est un cas d'étymologie populaire.

L'oubli de la forme première et l'obscurité imprécise de la voyelle du radical après la chute de la consonne médiane, ont ainsi amené la variété des formes prononcées da-an', do-an', dou-an'. La dernière prévaut, parceque commençant par le doo (orthographiquement $d u b h$ ) 'noir', elle offre un sens et une explication. Il n'en a pas été autrement dans les autres dialectes gaeliques, ceux d'Ecosse et de Man. Nous trouvons la même gamme de noms en Ecosse:

1) C'est-à-dire que la forme littéraire avec l'orthographe traditionnelle devrait venir après la forme populaire, et cela quand elle est ou connue, ou connaissable, ou supposable, et dans ce dernier cas avec un astérisque, comme lorsque les linguistes reconstruisent une forme non historique et par hypothèse. C'est un système que ne désapprouvera pas le $P$. Edmund Hogan, à ce que je vois par la préface de son utile Luibhleablrán, Dublin 1900: mais l'exemple qu'il y donne lui-même, dohough, dugh, n'est pas phonétique, puisque dans ces deux mots la consonne finale ne se prononce pas. 
damhan allaidh dans la traduction écossaise de la Bible publiée à Londres en 1807 par la Société Biblique (aux mêmes endroits que dans la Bible irlandaise de Bedel) dabhan-alluidh, dabhan-eallaich [autre graphie de l'épithète parceque la consonne finale ne se prononce pas], damhan-alluidh, et dubhan-alluidh, dans le Dictionarium Scoto-Celticum de la Highland Society, Edinburgh 1828, t. I, p. 323, 327 et 376; - mêmes formes dans le Facclair Gaidhlig (on Gaelic Dictionary) d'E. Macdonald, Herne Bay (Kent), 1902 (s. v.) - et dans A. R. Forbes, Gaelic Names of Beasts, Edinburgh 1905, p. 49 et 419.1)

Le gaelique de Man a une forme contractée d'où a disparu le suffixe -án, et qui donne l'impression de signifier seulement la 'noiraude' et il figure dans la longue liste des mots qui commencent par ce terme. C'est, dans l'horrible graphie du mannois, doo-oallee chez J. Kelly, Manx and English Dictionary, édition de J. I. Mosley, Douglas 1866, s. v., et doo-oalee dans Cregeen, Dictionary of the Manks Language, Douglas 1835, s. v.

Comme autres noms irlandais de l'araignée, nous trouvons dans les livres: figheadóir, dans Fournier d'Albe, English-Irish Dictionary, Dublin 1903, s. v. Spider; - de même figheadair, dans le Dictionarium Scoto-Celticum, t. II, p. 779 b. Le mot signifie simplement 'la fileuse' et traduit au moyen-âge le latin textor, voir Wh. Stokes, Irish Glosses, Dublin 1860, p. 34 et 126, no 1095.

Le dictionnaire anglais-irlandais de Foley donne aussi cui$\operatorname{deog}$ (p. 324a) dont je ne vois pas l'explication.

Il $y$ a certainement, du reste, bien des noms en existence pour désigner les araignées en Irlande, d'autant plus qu'il y a de nombreuses variétés de l'insecte. Ainsi je n'ai pu trouver nulle part de nom irlandais pour cette araignée des champs, à pattes fort longues, qu'on appelle en français faucheux, parceque dans sa démarche elle ressemble à un homme qui fauche. On l'appelle aussi en anglais de noms analogues, ?arvest man, haymaker, daddy long legs etc.: c'est le phalangium opilio des naturalistes. ${ }^{2}$ ) Comment l'appelle-t-on en Irlande? car elle y est indigène comme chez nous, et, comme chez nous, elle doit y avoir un nom populaire.

1) Cet ourrage de M. Forbes est de peu d'utilité, car il est écrit sans critique philologique et sans précision scientifique.

2) Voir Eug. Rolland, Faune populaire de la France, t. III, 1881, p. 244. 
IV.

Revenons à notre point de départ et à la question de sémantique que pose le nom ancien de l'araignée, damán allaid, 'le petit dam sauvage', plus exactement 'le petit cerf sauvage': Comment l'araignée a-t-elle pu être appelée du nom d'un mammifère quadrupéde? Car un nom analogue ne se rencontre guère ailleurs, sauf quelques exceptions que nous dirons tout-àl'heure. On n'en connait pas dans les langues celtiques de la famille britanniques ni dans les langues germaniques où le nom de l'araignée signifie en général 'la fileuse. Les noms romans dérivent - sauf exceptions locales et populaires du latin aranea, et le mot latin vient probablement lui-même du grec.

Il s'agit donc, dans l'ancien nom irlandais de l'araignée, d'une métaphore. La métaphore est un rapport où l'on voit, comme dans un éclair, certaine analogie des choses par un côté ou par un autre: le peuple, à cet égard, possède un esprit plus vif et plus poétique que le lettré. Damán allaid, ne l'oublions pas, est, à proprement parler, non pas un mot composé, mais un diminutif d'un terme existant déjà, dam allaid. Ce dernier nom désignait un animal bondissant, rapide, fuyant à l'approche ou à la vue de l'homme: l'araignée, aussi, fuit rapide et comme bondissante lorsque l'homme veut la saisir ou qu'elle l'aperçoit: ainsi l'araignée a du à sa démarche agile le nom de 'petit cerf sauvage', puisque déjà par le déterminant allaid 'sauvage', le mot dam avait, du sens de 'bœuf', passé à celui de 'cerf'.

La même idée s'est présentée en France, en plusieurs endroits, et elle a donné naissance à des noms populaires et locaux de l'espèce particulière d'araignée appelée, en français général 'le faucheux'. Je les cite d'après M. Eug. Rolland 1): Chièbe c. à d. chèvre; et tchiève-arine c. à d. chèvre-araignée les deux noms viennent de localités diverses du Berry. Cabro, c. à d. chèvre, dans le département des Basses-Alpes; Tsabromartelo, c. à d. chèvre-marteau ${ }^{2}$ ) dans le département du Puyde Dôme.

1) Faune populaire, T. XII (non encore publié), p. 140-141.

2) L'épithète de 'marteau' s'explique par une particularité du faucheur, sur laquelle voir Rolland, Faune, T. III, p. 244-245. 
En Irlande, l'araignée était nommée d'après le cerf: en France elle l'est quelquefois d'après la chèvre, animal tout aussi dissemblable dans l'ordre de la nature. Mais la dissemblance n'est pas plus grande ici qu'entre la chèvre et le petit crustacé décapode des naturalistes qu'on appelle à Paris crevelte (en anglais shrimp, en allemand garneele). Crevette est une forme normande et picarde de notre diminutif chevrette c. à d. 'petite chèvre' ainsi nommée des petits sauts que fait l'animal dans le sable du rivage.1) De là aussi pour le même crustacé le nom de sauterelle de mer, ou simplement sauterelle et d'autres noms analogues. ${ }^{2}$ ) Le sens originel du mot crevette est vivant sur nos côtes du Nord, puisque c'est simplement une forme dialectale, mais il ne l'est pas à Paris; et les Parisiens, à moins d'être philologues, ne pensent qu' au crustacé comestible, et non à la chèvre, quand ils entendent les marchandes au panier crier dans les rues: 'la crevette, fraiche et bonne! ma belle crevette!' L'etymologie est également ignorée, sauf des philologues, quand il s'agit de mots qui viennent, par métaphore, du nom de la chèvre, comme cabriole, cabriolet, se cabrer.

Ces rapprochements d'ordre psychologique, où l'on voit le nom de la chèvre donnée à une araignée et à un crustacé, aident à comprendre comment le nom du cerf a pu être donné, par métaphore, à l'araignée. Mais le nom irlandais damán allaid apporte un autre enseignement. Il y a mille ans il signifiait bien, pour ceux qui l'employaient 'le petit cerf sauvage': aujourdhui, par suite d'ume transformation, régulière en phonétique, son premier sens n'est plus sensible; et, l'étymologie populaire s'en mêlant, le nom signifie maintenant 'la noiraude du mur'. C'est ce qu'on pourrait appeler du métamorphisme étymologique. Et supposons que l'ancienne langue irlandaise ne nous ait pas laissé de document écrit, que l'on connaisse seulement la forme moderne duanalla: le sens moderne paraitra la véritable étymologie, et ce sera chose certaine et indiscutable pour les philologues ... N'estce pas ainsi que l'on raisonne avec les langues de l'antiquité dont nous ne connaisons pas les périodes antérieures? Crede parum!

1) Dictionnaire général de la langue française par Hatzfeld, Darmesteter et Thomas, s. v. crevette et chevrette.

2) S. Rolland, Faune populaive, T. XII, p. 99 et III (1881), p. 228. 


\section{John K'eogh précurseur de la phonétique.}

Le nom de Keogh est généralement la transcription anglaise du nom irlandais Mac Eochaidh. ${ }^{1}$ ) On a vu dans l'article précédent comment j'écrivais le nom de John K'eogh: c'est parceque l'auteur écrit ainsi son propre nom dans sa Botanologia et dans sa Zoologia.

Evidemment c'est pour indiquer que dans la prononciation de son nom il faut bien distinguer la consonne initiale, et presque la séparer de la voyelle qui suit. C'est un exemple de ce que les linguistes appellent un ' $\mathrm{k}$ palatal', celui qu'on entend quelquefois à Dublin dans le mot car quand un cocher vous interpelle dans la rue: 'Do you want a kyar, your Honour?'

Le $\mathrm{K}^{\prime}$ de John K'eogh est donc la plus ancienne notation connue du $\mathrm{K}$ palatal irlandais, et cela par le même sigle ( $\left.\mathrm{K}^{\prime}\right)$ que devaient inventer plus tard les linguistes modernes.

L'orthographe personnelle de J. K'eogh a été respectée par A. Webb, Compendium of Irish Biography Dublin, 1878, p. 272. O'Donovan n'en avait pas tenu compte, comme on peut voir dans ses Topographical Poems, Dublin, 1862, p. LxxxIII. Mais il est surprenant que dans la préface de son Luibhleabhrán Dublin, 1900, le P. Edmund Hogan, lui-même un grammairien, écrive systématiquement ce nom: $K^{\prime} \operatorname{Eogh}$ (p. v, IX et xir).

Une lettre majuscule a sa raison d'être dans des noms comme 0'Reilly ou M'Millan par ce qu'elle commence un mot nouveau, et qu'on prononce le préfixe $0^{\prime}$ ou $\mathbf{M}^{\prime}$ (Mac) comme un phonème qui peut se distinguer: mais le $K^{\prime}$ de $K^{\prime}$ eogh ne forme ni un mot ni un phonème, et il n'a qu'une valeur de signe phonétique, non étymologique. En imaginant cette graphie contre-nature $K^{\prime}$ Eogh, le P. Hogan a été instinctivement trompé par l'analogie usuelle des noms en 0 ' et en $M$ '.

L'analogie mène souvent à des erreurs. En voici une que j'apprenais récemment de $M$. Diverrès et qui mérite d'être notée. On sait qu'en Bretagne pour abréger dans l'écriture on écrivait autrefois la syllabe Ker, si fréquente comme préfixe de noms de lieu et, par suite, de noms d'homme, en un sigle: une lettre $K$

1) La graphie $K$ 'eogh comme nom de famille existe encore, car je la trouve - en même temps que Cuhy, Kehoe, Keoghoe, Keoghy, Keough, Kough et Mc Cahugh - dans R. E. Matheson, Varieties and Synonyms of Surnames and Christian Names in Ireland, Dublin, 1901, p. 47. 
dont la branche de droite, un peu allongée, est traverseee par une barre. Le $K$ barré se lisait donc régulièrement ker, et soit dit en passant, ce sigle d'abréviation donne quelquefois lieu à des difficultés avec des employés de l'état-civil, ignorants de la Bretagne ou du passé. Or il y a à Lorient une rue Kleber; naturellement, les gens du peuple ignorent ce héros que l'Alsace a donné à la France, et ils disent - ou, du moins, disaient habituellement il y a une dizaine d'années - 'la rue Kerleber', prenant ce nom étranger pour un nom breton écrit à la bretonne.

Pour en revenir à John K'eogh, c'est un précurseur de la science phonétique si en honneur aujourd'hui: il m'a paru convenable de le rappeler au passage.

Paris.

Henri Gaidoz. 